\title{
Enhanced seeded free electron laser performance with a "cold" electron beam
}

\author{
G. Penco $\odot,{ }^{1}$ G. Perosa, ${ }^{2}$ E. Allaria $\odot,{ }^{1,3}$ S. Di Mitri®,${ }^{1,2}$ E. Ferrari, ${ }^{4}$ L. Giannessi, ${ }^{1,5}$ \\ S. Spampinati, ${ }^{1}$ C. Spezzani, ${ }^{1}$ and M. Veronese ${ }^{1}{ }^{1}$ \\ ${ }^{1}$ Elettra-Sincrotrone Trieste S.C.p.A., S.S. 14-km 163.5 in AREA Science Park 34149 Basovizza, \\ Trieste, Italy \\ ${ }^{2}$ Università degli Studi di Trieste, Dipartimento di Fisica, Piazzale Europa 1, Trieste, Italy \\ ${ }^{3}$ Deutsches Elektronen-Synchrotron DESY, Notkestrasse 85, 22607 Hamburg, Germany \\ ${ }^{4}$ Paul Scherrer Institut, Large Research Facilities (GFA) Forschungsstrasse 111, \\ 5232 Villigen PSI Schweiz. \\ ${ }^{5}$ INFN, Laboratori Nazionali di Frascati, Via E. Fermi 40, 00044 Frascati, Roma
}

(Received 16 September 2020; accepted 10 December 2020; published 28 December 2020)

\begin{abstract}
FERMI is a seeded free electron laser (FEL) based on the high gain harmonic generation (HGHG) scheme and has been generating intense and fully coherent extreme ultra-violet and soft x-ray pulses for several years. The high-degree performance of the FEL leans on a high brightness electron beam, with small transverse emittance $(\sim 1 \mu \mathrm{m})$ and a peak current of about 700-800 A. The main constraint on lasing at high harmonics of the seed is low electron time-slice energy spread. The optimization of the photoinjector and of some linac parameters has allowed a reduction of the relative slice energy spread to the level of few times of $10^{-5}$. With these new conditions, the FEL can be operated without the need of a laser heater to suppress micro bunching instabilities and this "cold" beam has allowed generation of extreme UV pulses with pulse energy exceeding a mJ, and with peak power of about $10 \mathrm{GW}$. We describe the electron beam characterization and the FEL performance improvement, including the extension of the range of harmonics of the seed which can be amplified, up to the twenty-fifth harmonic, i.e., $10 \mathrm{~nm}$.
\end{abstract}

DOI: 10.1103/PhysRevAccelBeams.23.120704

\section{INTRODUCTION}

Free electron lasers (FELs) can exceed by about 10 orders of magnitude the peak brightness of existing synchrotron sources. Their unique capability of producing very intense and short pulses, at wavelengths ranging from tens of $\mathrm{nm}$ to sub Ångström [1,2], are opening new scientific possibilities and enabling the access to new matter states [3]. Most of these FELs amplify the stochastic electronic noise of a high current beam passing through a long magnetic undulator. Such a self-amplified spontaneous emission (SASE) mode of operation, does not guarantee the generation of longitudinally fully coherent pulses. Each FEL pulse is typically composed of a burst of many short spikes, with random phases and amplitudes [4,5]. Longitudinal coherence can be improved by injecting a coherent seed radiation pulse to initiate the FEL amplification process. Several schemes implementing the seed have been proposed and demonstrated. These can be

Published by the American Physical Society under the terms of the Creative Commons Attribution 4.0 International license. Further distribution of this work must maintain attribution to the author(s) and the published article's title, journal citation, and DOI. classified as externally seeded configurations [6-9] and self-seeded configurations [10-14].

FERMI [15] is a FEL based on the high gain harmonic generation scheme (HGHG) [6] where an optical seed is frequency multiplied and amplified, preserving some of the original pulse coherence properties. This scheme allows generation of temporally and spatially coherent pulses and control of the slowly varying phase along the FEL pulse [16]. Coherence is also preserved at higher orders [17], and it has been exploited in coherent control experiments as [18] and for the generation of multiple-color multiple pulses with a well determined and controlled phase relation in the EUV and soft X-rays [18-23].

This progress has stimulated a search for the ultimate limits of the HGHG scheme in the generation of high harmonics. The harmonic conversion efficiency of HGHG decreases at high harmonics (shorter wavelengths) [6,7] and the quality of the pulses is increasingly affected by the electron beam defects, such as phase space distortions $[24,25]$ or microbunching instability. The latter is induced by the collective self-amplification of nonuniformities in the electron longitudinal distribution [26,27]. In fact, an initial density modulation in the beam current profile is converted into an energy modulation by longitudinal space charge forces. The energy modulation is in turn converted 
into density modulation when the electrons pass through dispersive sections such as magnetic compressors, leading to amplification of the initial modulation. The suppression of microbunching instability is of critical importance to avoid structures in the electron beam phase-space that affect the longitudinal coherence of FEL pulses.

Microbunching instability gain is sensitive to the slice energy spread $\left(\sigma_{E}\right)$. A laser heater (LH) device [28] is used to properly increase $\sigma_{E}$, damping the instability growth [29]. The use of a LH has shown the capability to improve the HGHG performances in terms of longitudinal coherence. Unfortunately, the LH-induced $\sigma_{E}$ increment prevents an efficient harmonic conversion, so that a trade-off between microbunching damping and FEL amplification at high harmonics has to be found. During routine operation of FERMI, a modest level of heating is sufficient to suppress the microbunching. The typical $\sigma_{E}$ at the undulator entrance is of the order of $100-150 \mathrm{keV}[8,9]$, i.e., $\delta=\sigma_{E} / E \approx 10^{-4}$. In these conditions, the single stage HGHG in FEL-1 can be operated for users up to harmonic $13-15$ of the UV seed (260-240 nm), reaching a wavelength range $16-20 \mathrm{~nm}$ [8].

Among the various seeding schemes recently proposed [7,30,31], echo enabled harmonic generation [7] allows improvement of the high harmonic conversion efficiency and a reduction of the sensitivity to electron beam phase space modulations [24,25,28-32]. However, also the performances of FERMI operating in HGHG can be also improved, if a solution to suppress microbunching instability without increasing the slice energy spread with a LH is found.

In this work, we report recent results obtained under conditions allowing transport of the electron beam up to the FERMI FEL-1 undulator without significant gain of the microbunching instability. In the following, we will focus on the improved performance of the FEL when such an electron beam is used for generating coherent HGHG radiation.

The first part of the paper is dedicated to the electron beam characterization, showing the measurements of the electron slice energy spread obtained by setting the FEL in SASE optical klystron mode and optimizing the dispersive section strength [33]. In the second part, we focus on the FEL performance obtained with this "cold" beam, both in terms of spectral purity and intensity at high harmonics.

\section{ELECTRON BEAM}

The FERMI electron beam is generated from a copper photocathode illuminated by an intense UV laser at $260 \mathrm{~nm}$ (namely, photoninjector laser, PIL) and immediately accelerated in a 1.6-cell rf gun cavity (S-band) up to about $5 \mathrm{MeV}$. The typical charge per bunch is in the range 600$700 \mathrm{pC}$, with a repetition rate of $10 \mathrm{~Hz}$ or $50 \mathrm{~Hz}$ [34].

Temporal modulation in the shape of the PIL can introduce modulations in the bunch current profile. In fact, the PIL is obtained from a 100 fs-long, large bandwidth pulse, that is stretched to a few ps. As a consequence, small modulations with periodicity of tens of micrometers (over a bunch length of about $2 \mathrm{~mm}$ ) can be impressed onto the electron temporal profile and amplified by the microbunching instability.

In September 2019, a new copper cathode was installed and a careful optimization was done to preserve its transverse uniformity. The photoinjector laser was upgraded for an improved transverse uniformity and smoothness in the longitudinal direction. Furthermore, in order to limit the generation of undesired temporal modulation, the PIL bandwidth has been reduced by $30 \%$ by decreasing the bandwidth of the fundamental infrared pulses by about $20 \%$, followed by the use of a combination of thicker second and third harmonic crystals in the UV generation setup. As a result of this upgrade, at the injector exit $(\sim 100 \mathrm{MeV})$, a projected emittance of about $1.2-1.3 \mu \mathrm{m}$ was measured in both planes for a bunch with a charge in the range $600-700 \mathrm{pC}$ and a FWHM length of about 8 ps. The beam is routinely compressed to a nominal peak current of $\sim 700-800 \mathrm{~A}$ at about $300 \mathrm{MeV}$ by means of a single compressor (BC1), as it has been proven that it is less affected by microbunching growth than the double compression scheme [35].

Concerning the beam optics, the typical strategy adopted to match the beam optics in the FERMI linac consisted of imposing predetermined Twiss parameters in three particular matching points: at the injector exit, after the first compressor BC1, and the end of the linac (see [36]), and then setting a quasi-FODO lattice in between them. This strategy has been recently revised. We increased the beam beta functions at the matching points, also relaxing the conditions to match to a beam waist at these positions. The beam optics in the spreader was also investigated to better control the residual dispersion and chromaticity after the spreader [37]. Moreover, this new tool allows exploration of different beam optics solutions in order to mitigate the microbunching instability growth by avoiding excessively strong focusing, i.e., high charge density, at lower energy. The combined effect of these changes led to the transport of a beam with minimal microbunching instability amplification and with a substantially lower energy spread, that will be referred to as the "cold" beam.

\section{A. Characterization of the electron beam}

Measurements of the time-slice energy spread of the electron bunch are routinely performed by using an $\mathrm{rf}$ deflector placed at the end of the linac, in combination with the energy spectrometer [38]. The measurement resolution depends on several parameters (beam optics, rf deflecting voltage, beam energy). In the specific case of FERMI, considering a beam energy in the range $1.0-1.5 \mathrm{GeV}$, the minimum energy spread value that can be resolved is about $100 \mathrm{keV}$ [29]. The cold beam produced at FERMI after the 
mentioned changes has a slice energy spread well below this value and an alternative method is necessary to measure this parameter. FERMI FEL-1 can be operated in SASE as a high-gain optical klystron $[33,39,40]$. According to $[39,41,42]$ the maximum FEL intensity is obtained when:

$$
\frac{R_{56} k_{r} \sigma_{E}}{E}=1
$$

where $R_{56}$ is the momentum compaction of the dispersive section, $k_{r}=\frac{2 \pi}{\lambda_{r}}$ and $\lambda_{r}$ is FEL output wavelength. The SASE optical klystron is therefore a diagnostic tool to measure the beam energy spread that can be calculated from the value of the dispersion corresponding to the maximum FEL intensity.

The modulator and the radiator of FEL-1 were tuned to be resonant at $\lambda=31.9 \mathrm{~nm}$ and the dispersive section strength between them has been scanned. Figure 1 shows the FEL pulse energy measured as a function of the dispersive section $R_{56}$ for different values of the laser heater intensity, and for a beam energy $E=1.32 \mathrm{GeV}$. In order to have a high SASE intensity we initially compressed the electron beam more than in the nominal case reaching a peak current $I_{p} \sim 1.1 \mathrm{kA}$.

The first indication of a reduced microbunching instability gain and of a low energy spread, is given by the fact that the best FEL performance, in terms of pulse energy, is obtained in the absence of the laser heater. This differs from what was observed in the past for a similar FEL configuration (see [33]). On that occasion, the FEL pulse energy was maximized for a low (but not zero) intensity of the laser heater, with an $R_{56}$ value of $75 \mu \mathrm{m}$ (see vertical dotted line on the inset of Fig. 1). In contrast, in the present situation the maximum FEL pulse energy decreases and shifts toward smaller values of $R_{56}$ when the laser heater power is increased. This is a clear indication of a progressive and

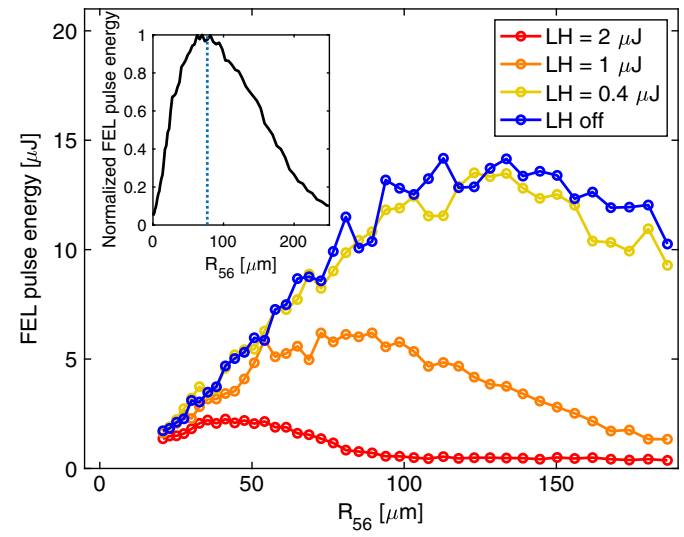

FIG. 1. FEL pulse energy at $31.9 \mathrm{~nm}$ versus the dispersive section $R_{56}$, for different values of the laser heater energy. Inset reports, for comparison, the FEL normalized pulse energy at $32.1 \mathrm{~nm}$ versus $R_{56}$ from previous experiments [33]. monotonic growth of the slice energy spread, without the typical minimum for nonzero heating. Similar measurements have been repeated for the nominal FERMI compressed beam, $I_{p} \sim 0.8 \mathrm{kA}$, and for higher compression, $I_{p} \sim 1.3 \mathrm{kA}$.

Applying Eq. (1), $\sigma_{E}$ was retrieved in the mentioned cases and the results are summarized in Fig. 2. For the nominal case $\left(I_{p} \sim 0.8 \mathrm{kA}\right)$, a minimum $\sigma_{E}$ of about $40 \mathrm{keV}$ was measured, a value much lower than what was usually obtained. Also in the strong compression case $\left(I_{p} \sim 1.3 \mathrm{kA}\right), \sigma_{E}$ is minimized with the laser heater off. This suggests that no microbunching structures have been amplified along the machine, at least to a level that affects the energy spread. In terms of energy spread mitigation, the laser heater is actually not required with this electron beam condition.

As has been reported in the literature [30], the $\sigma_{E}$ parameter is of paramount importance. For a seeded FEL as FERMI based on the HGHG scheme, in order to be able to lase efficiently at a harmonic $h$ of the seed laser frequency, the following condition has to be met:

$$
\frac{\sigma_{E}}{E}<\delta_{\mathrm{FEL}} \sim \frac{\rho}{2 \sqrt{\left(h^{2}+1\right)}}
$$

where $\rho$ is the FEL parameter, i.e., Pierce parameter [43].

Figure 3 shows the maximum tolerable relative energy spread $\delta_{\mathrm{FEL}}$ calculated for FERMI FEL-1, from $40 \mathrm{~nm}$ to $10 \mathrm{~nm}$, according to the inequality (2) that provides exponential gain. In the past, the FERMI FEL-1 gain suffered a strong reduction for harmonics $(h)$ larger than 13-15, indicating a slice energy spread of the order of $100-150 \mathrm{keV}$, in agreement with the past energy spread measurements $[8,29,33]$. A beam slice energy spread of $40 \mathrm{keV}$ fulfills the condition (2) up to harmonic $26(\lambda=10 \mathrm{~nm}), \frac{\sigma_{E}}{E}<3 \times 10^{-5}$, for a beam energy of

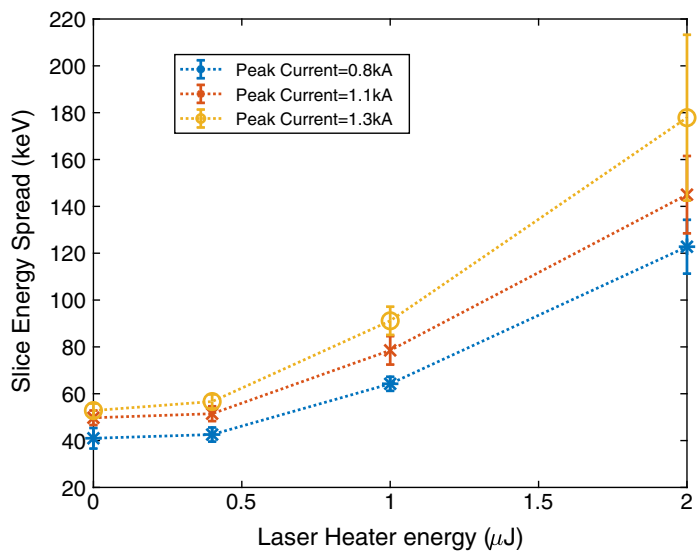

FIG. 2. Measured time-slice energy spread obtained from Eq. (1) applying the FEL versus $R_{56}$ scan as reported in Fig. 1. The error bars refer to a confidence interval of $68 \%$ in evaluating the optimum value of $R_{56}$. 


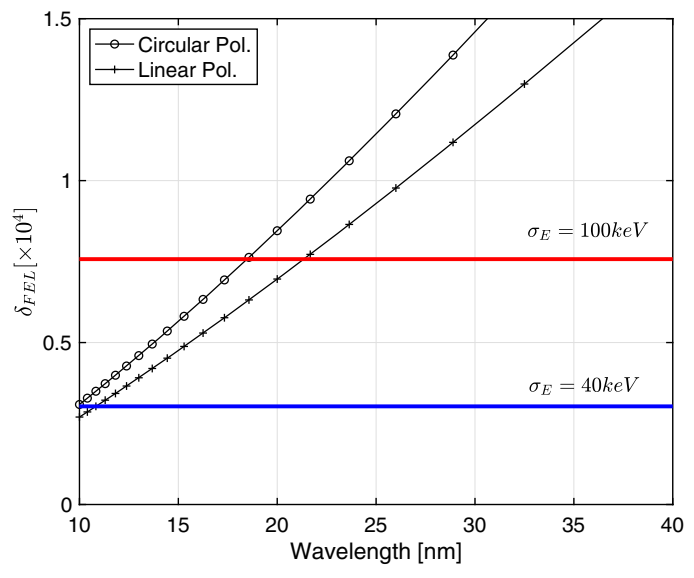

FIG. 3. The maximum $\delta_{\text {FEL }}$ versus the FEL output wavelength calculated for FERMI FEL-1 undulators tuned in circular and linear polarization, according to Eq. (2). The standard FERMI case $\left(\sigma_{E}=100 \mathrm{keV}\right)$ and the actual condition $\left(\sigma_{E}=40 \mathrm{keV}\right)$ are indicated with horizontal lines, assuming a beam energy of $1.32 \mathrm{GeV}$.

$1.32 \mathrm{GeV}$. This condition corresponds to the measured value in the reported conditions, and stimulated an investigation of the FEL performances.

\section{FEL PERFORMANCE AT FERMI}

At FERMI, the LH has been exploited to control periodic structures in the electron beam, in order to generate stable spectral sidebands, i.e., multicolor FEL pulses, or to shift the lasing frequency with respect to an integer harmonic of the seed [44], but its main application is the suppression of the microbunching instability $[29,45]$. The LH is considered essential in the optimization of the FEL aiming at maximizing the FEL intensity while preserving a single moded, gaussian-shaped, stable from pulse to pulse, radiation spectrum. The impact of the $\mathrm{LH}$ on the FEL performance depends upon several parameters including the use of bunch compressor and the original content of microbunching structures and their amplification [46]. At FERMI, the absolute advantage provided by the $\mathrm{LH}$, in terms of FEL intensity and spectral purity, has not been constant over the years, but a small amount of LH energy has been always necessary to optimize the FEL output.

A typical case is represented in Fig. 4, where the FEL intensity [Fig. 4(a)] and the FEL spectrum [Fig. 4(b)] at $22.5 \mathrm{~nm}(h=11)$ are reported as a function of the LH pulse energy controlled through an optical attenuator. When the LH energy is close to zero, the FEL presents a multispike spectrum and low pulse energy. It is necessary to increase the LH energy to about $1 \mu \mathrm{J}$ to get rid of the microbunching instability and maximize the FEL intensity with a "clean" spectrum.

After the recent changes mentioned in Sec. II, we observed a consistent reduction of the LH energy required to reach such a condition. Figures $4(\mathrm{~d}-\mathrm{f})$ show the same
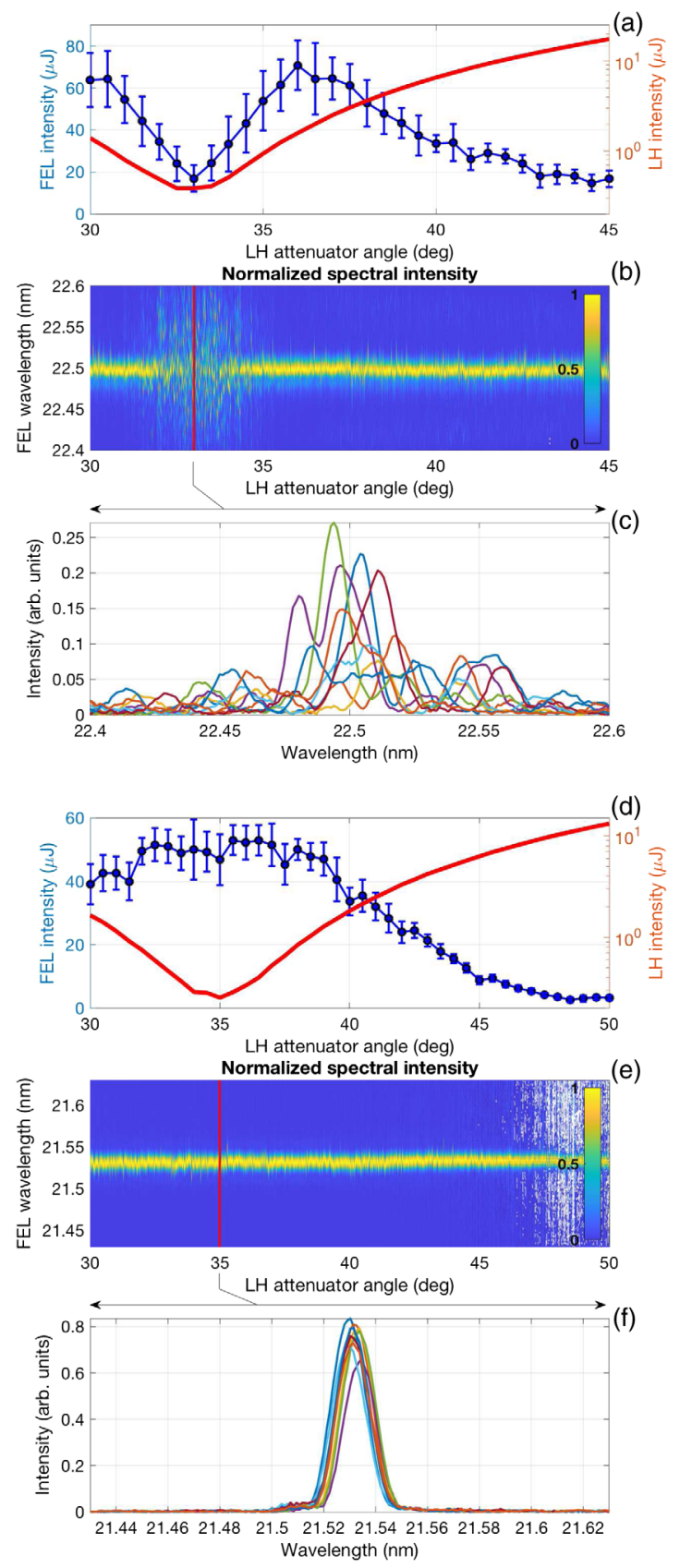

FIG. 4. FEL performance versus laser heater for a standard electron beam in 2018 (a,b,c) and for the current one (d,e,f). FEL intensity (blue curve) and laser heater intensity (red curve) (a,d) are plotted as a function of the LH attenuator angle. FEL spectra versus LH attenuator angle are reported in $(b, e)$ in false color scale (normalized for the maximum intensity value). Few spectra for the standard case (c) and for the actual case (f) have been randomly selected for the minimum value of the $\mathrm{LH}(\sim 0.2 \mu \mathrm{J})$.

measurements in the new configuration: the FEL spectrum is a single line without evidence of microbunching induced sidebands, and the maximum FEL intensity is obtained at the minimum LH intensity. Apparently, the LH has the effect of increasing the electron slice energy spread and consequently, reducing the FEL gain and the output 
intensity. This is consistent with the measurements reported in Fig. 2, where the minimum slice energy spread was observed for the LH at zero intensity.

Moreover, even in the case of the LH completely off, as shown in Fig. 5, the FEL at $\sim 21 \mathrm{~nm}$ is characterized by a shot-to-shot power stability of $\sim 10 \%$ (rms), with a bandwidth of $7 \times 10^{-4}$ (fwhm) averaged over 1800 shots. A small fraction of shots $(\sim 10 \%)$ have been filtered out as they were associated with a large jitter of the relative arrival time between seed and electron bunch, as measured by the bunch arrival monitor [47]. The measured spectral bandwidth corresponds to a Fourier limited pulse duration $\delta_{t}^{(\mathrm{fel})}$ of about $45 \mathrm{fs}$ (fwhm). This is in agreement with the expected pulse duration (51 fs) calculated from a seed pulse of about $100 \mathrm{fs}$ (fwhm) at $260 \mathrm{~nm}$, according to the rule $\delta_{t}^{(\mathrm{fel})}=7 / 6 \delta_{t}^{\text {(seed) }} / h^{1 / 3}$, where $h=12$ is the harmonic order [48]. The residual difference may be due to a small chirp of the seed laser pulse that causes an increase of the time bandwidth product of the FEL pulse. Note also that the scaling relation with the seed pulse duration represents a rough estimate, since the effective pulse duration depends on the level of saturation reached by the amplifier. In fact, far away from the saturation the $\delta_{t}^{(\mathrm{fel})}$ scales as $\delta_{t}^{\text {(seed) }} / h^{1 / 2}$.

In presence of time-dependent energy modulation along the bunch, the FEL wavelength output is slightly shifted shot-to-shot according to the temporal jitter between seed and electrons that is about $50 \mathrm{fs}$ [49]. However, in Fig. 5 the shot-to-shot wavelength stability (rms $\sim 8 \times 10^{-6}$ ) is anyway two order of magnitude smaller than the FEL bandwidth, and this is another important signature that the beam has no microbunching structures.

It is worthwhile mentioning that because of the low resolution, a measure of the electron longitudinal phase space performed with rf-deflector and energy spectrometer
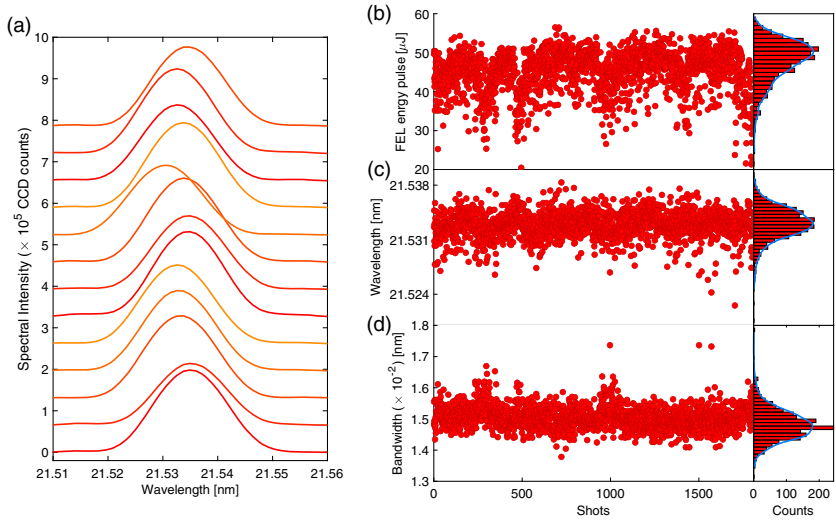

FIG. 5. Characterization of FEL pulses at $h=12(\sim 21 \mathrm{~nm})$ in linear horizontal polarization. Random selection of individual spectra (a) and statistical analysis over 1800 shots showing energy per pulse (b) central wavelength (c) and rms spectral bandwidth evolution (d). Few shots $(\sim 10 \%)$ have been removed from the analysis due to very different e-beam parameters. does not show any difference in terms of microbunching content between this cold beam and the previous situation. Conversely, this difference is highlighted by the performance of the FEL operating at high harmonics in HGHG mode.

The use of a cold electron beam (with $\sigma_{E} \sim 40 \mathrm{keV}$ ) without microbunching structures at the undulator entrance provides further advantages. As shown in Fig. 3, the low slice energy spread enables the amplification of shorter wavelengths, such as $14 \mathrm{~nm}(h=18)$. FEL operation in this wavelength range was reported in [50], but with a substantially lower pulse energy, and implementing an harmonic cascade scheme. In the present conditions, more than $10 \mu \mathrm{J}$ per pulse were measured, with a relative (fwhm) FEL bandwidth of about $6.4 \times 10^{-4}$, as reported in Fig. 6 . The measured FEL bandwidth corresponds to a Fourier limited pulse of about $32 \mathrm{fs}$ (fwhm), in agreement with the aforementioned scaling law [48] that foresees an FEL pulse duration ranging from 24 fs to 45 fs depending upon the level of saturation. Note that data reported in Fig. 6 refer to linearly polarized light. More than a factor two of power increase is expected in the case of a circularly polarized undulator.

We measured the FEL pulse energy at various wavelengths, in particular from $35 \mathrm{~nm}(h=7)$ down to $10 \mathrm{~nm}$ $(h=25)$. The measurements down to $20 \mathrm{~nm}$ were carried out with the FERMI intensity monitor [51]. At shorter wavelengths, the pulse energy was estimated from the intensity of the spectrometer camera image [52], crosscalibrated with the intensity monitor. An average energy of about $0.5 \mu \mathrm{J}$ at $10 \mathrm{~nm}$ was estimated, with shots up to $1.5 \mu \mathrm{J}$. The results are shown in Fig. 7. The red continuous line corresponds to the standard FEL pulse energy. The performances of the FEL with the cold beam
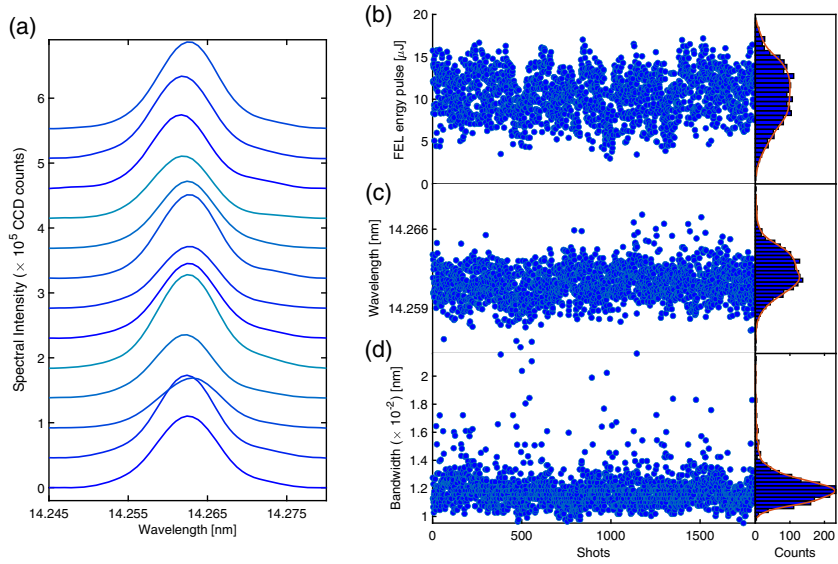

FIG. 6. Characterization of FEL pulses at $h=18$ (i.e., $\sim 14 \mathrm{~nm}$ ) in linear horizontal polarization. Random selection of individual spectra (a) and statistical analysis over 1800 shots showing energy per pulse (b) central wavelength (c) and rms spectral bandwidth evolution (d). Few shots $(\sim 10 \%)$ have been removed from the analysis due to very different e-beam parameters. 


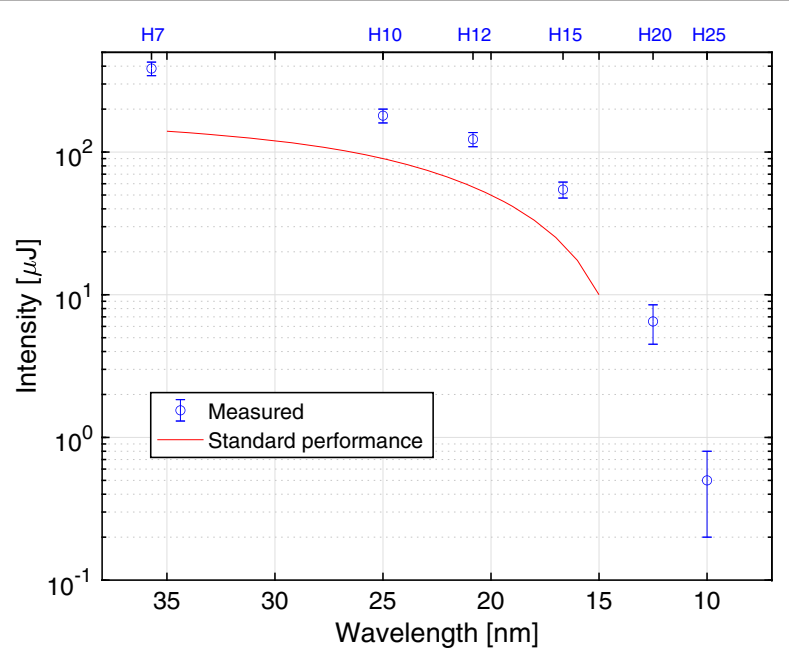

FIG. 7. Average energy per pulse (blue dots) measured for FEL-1 in circular polarization from $h=7(\sim 35 \mathrm{~nm})$ down to $h=25(\sim 10 \mathrm{~nm})$. Error bars correspond to the standard deviation over hundred of shots. Red line corresponds to the standard guaranteed FERMI FEL-1 energy as reported in [53].

significantly exceed the standard one in the whole range. Moreover, in this configuration we could measure a good signal down to $10 \mathrm{~nm}$, thus extending the lasing range of FERMI FEL-1.

The lower beam energy spread can be also exploited to increase the beam current with a stronger compression. We operated the FEL with a bunch compressed by a factor 15 corresponding to a final peak current of about $1.3 \mathrm{kA}$. After optimization at $h=7$ (i.e., $37 \mathrm{~nm}$ ) in circular polarization, the FEL intensity reached several hundreds of $\mu \mathrm{J}$. A taper of the final undulators and a careful optimization of the seeding parameters allowed to measure FEL pulses exceeding $1 \mathrm{~mJ}$ for the first time at FERMI. Taking into account the scaling law already mentioned [48], one can estimate an FEL pulse length of about $60 \mathrm{fs}$, and therefore an FEL peak power at $37 \mathrm{~nm}$ of about $16 \mathrm{GW}$. Figure 8(a) reports the FEL amplification (blue line) along the undulator chain, together with the energy offsets corresponding to the undulator taper (red line).

It must be noted that despite the stronger compression, only a small fraction of the bunch charge participates to lasing in a seeded FEL where the seed pulse is much shorter than the electron bunch. The energy offset of the resonance of the last radiator was $15 \mathrm{MeV}$, corresponding to more than $1 \%$ of energy loss for the emitting electrons. Considering a peak current of $1.3 \mathrm{kA}$ and the mentioned energy loss, one can estimate an FEL peak power of about $20 \mathrm{GW}$, consistent with the previous estimation. Evolution of the FEL spectra as a function of the number of undulators is reported in Fig. 8(b). For each undulator setting, spectra are normalized to the corresponding maximum. This representation of the data allows identification of possible spectral structures even in case of weak spectra. Despite the very high compression and the fact that the laser heater

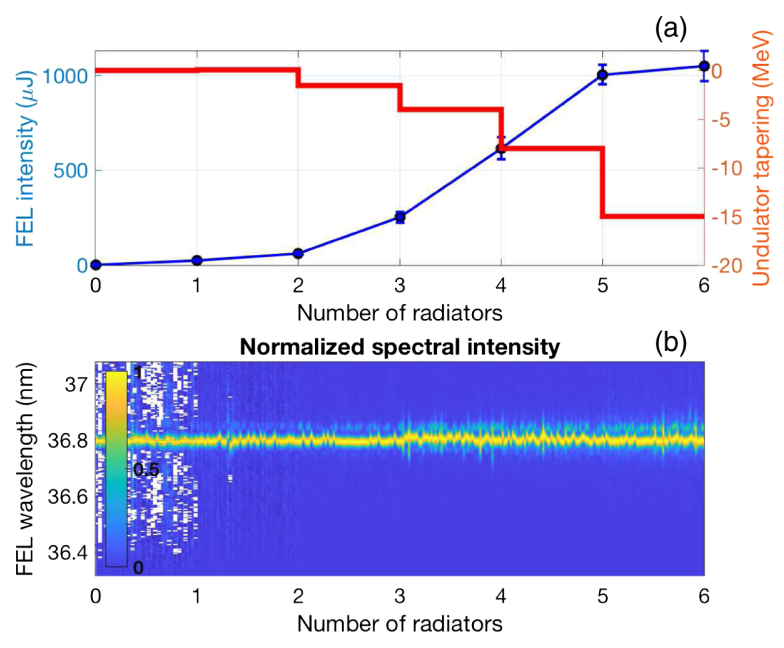

FIG. 8. FEL gain curve for an highly compressed beam $(\sim 1.3 \mathrm{kA})$ at $h=7(\sim 37 \mathrm{~nm})$ in circular polarization. (a) FEL energy growth as a function of the number of resonant undulators (blue curve) and applied tapering (red curve). (b) Normalized spectra along the gain curve.

was offline, FEL pulses show very stable and narrow spectra, without undesired features. The small sideband toward longer wavelength is expected to be the result of a combination between resonance tuning and FEL saturation.

\section{CONCLUSION}

We have experimentally demonstrated at FERMI, the possibility to generate, accelerate and compress above $1 \mathrm{kA}$, an electron bunch with the nominal charge in the range $600-700 \mathrm{pC}$ with an unprecedented small slice energy spread. This fact allowed significant improvement of the seeded FEL performance of FERMI. The output power increased by more than a factor two in the nominal range of operation of FEL-1. The standard operation range of the FEL was extended to higher harmonics, obtaining about $1 \mu \mathrm{J}$ at $h=25$ (i.e., $10 \mathrm{~nm}$ ). The bunch, virtually free of microbunching heating has also allowed the FEL operation at higher compression, producing $\mathrm{mJ}$-level pulses at $37 \mathrm{~nm}$. This result represents a foundation to improve the capabilities of quantum optics experiments and nonlinear optics experiments with fully coherent EUV pulses. A number of the related techniques have been pioneered at FERMI; applications include precision two-photon spectroscopy [54,55], coherent control $[18,56]$, pulse shaping $[21,23]$, and four-wave mixing $[57,58]$.

\section{ACKNOWLEDGMENTS}

Authors would like to thank all the FERMI Team for the support provided during the machine tuning and optimization. In particular they are grateful to Fatma Iazzourene for the development of the code to match the beam optics along the linac and the undulator line in a more 
flexible way, to Giovanni De Ninno for useful discussions on the experimental results and Giulio Gaio for the implementation of feedback and optimization tool that has been guaranteering a stable operation.

[1] P. R. Ribič and G. Margaritondo, Status and prospects of Xray free-electron lasers (X-FELs): A simple presentation, J. Phys. D 45, 213001 (2012).

[2] C. Bostedt, S. Boutet, D. M. Fritz, Z. Huang, H. J. Lee, H. T. Lemke, A. Robert, W. F. Schlotter, J. J. Turner, and G. J. Williams, Linac coherent light source: The first five years, Rev. Mod. Phys. 88, 015007 (2016).

[3] C. Pellegrini, A. Marinelli, and S. Reiche, The physics of X-ray free-electron lasers, Rev. Mod. Phys. 88, 015006 (2016).

[4] R. Bonifacio, C. Pellegrini, and L. M. Narducci, Collective instabilities and high-gain regime in a free electron laser, Opt. Commun. 50, 373 (1984).

[5] A. M. Kondratenko and E. L. Saldin, Generation of coherent radiation by a relativistic electron beam in an undulator, Part. Accel. 10, 207 (1980), http://cds.cern.ch/ record/1107977.

[6] L. H. Yu, Generation of intense uv radiation by subharmonically seeded single-pass free-electron lasers, Phys. Rev. A 44, 5178 (1991).

[7] G. Stupakov, Using the Beam-Echo Effect for Generation of Short-Wavelength Radiation, Phys. Rev. Lett. 102, 074801 (2009).

[8] E. Allaria et al., Highly coherent and stable pulses from the FERMI seeded free-electron laser in the extreme ultraviolet, Nat. Photonics 6, 699 (2012).

[9] E. Allaria et al., Two-stage seeded soft-X-ray free-electron laser, Nat. Photonics 7, 913 (2013).

[10] G. Geloni, V. Kocharyan, and E. Saldin, A novel selfseeding scheme for hard X-ray FELs, J. Mod. Opt. 58, 1391 (2011).

[11] G. Geloni, Self-seeded free-electron lasers, in Synchrotron Light Sources and Free-Electron Lasers, edited by E. Jaeschke, S. Khan, J. Schneider, and J. Hastings (Springer, Cham, 2016).

[12] J. Amann et al., Demonstration of self-seeding in a hard-Xray free-electron laser, Nat. Photonics 6, 693 (2012).

[13] D. Ratner et al., Experimental Demonstration of a Soft XRay Self-Seeded Free-Electron Laser, Phys. Rev. Lett. 114, 054801 (2015).

[14] I. Inoue et al., Generation of narrow-band X-ray freeelectron laser via reflection self-seeding, Nat. Photonics 13, 319 (2019).

[15] E. Allaria et al., The FERMI free-electron lasers, J. Synchrotron Radiat. 22, 485 (2015).

[16] G. De Ninno et al., Single-shot spectro-temporal characterization of XUV pulses from a seeded free-electron laser, Nat. Commun. 6, 8075 (2015).

[17] O. Y. Gorobtsov et al., Seeded X-ray free-electron laser generating radiation with laser statistical properties, Nat. Commun. 9, 4498 (2018).

[18] K. C. Prince et al., Coherent control with a short-wavelength free-electron laser, Nat. Photonics 10, 176 (2016).
[19] A. Wituschek, Tracking attosecond electronic coherences using phase-manipulated extreme ultraviolet pulses, Nat. Commun. 11, 883 (2020).

[20] D. Gauthier, P. R. Ribič, G. De Ninno, E. Allaria, P. Cinquegrana, M. B. Danailov, A. Demidovich, E. Ferrari, and L. Giannessi, Generation of Phase-Locked Pulses from a Seeded Free-Electron Laser, Phys. Rev. Lett. 116, 024801 (2016).

[21] P. K. Maroju et al., Attosecond pulse shaping using a seeded free-electron laser, Nature (London) 578, 386 (2020).

[22] M. Di Fraia et al., Complete Characterization of Phase and Amplitude of Bichromatic Extreme Ultraviolet Light, Phys. Rev. Lett. 123, 213904 (2019).

[23] D. Gauthier et al., Chirped pulse amplification in an extreme-ultraviolet free-electron laser, Nat. Commun. 7, 13688 (2016).

[24] E. Hemsing, M. Dunning, C. Hast, T. O. Raubenheimer, S. Weathersby, and D. Xiang, Highly coherent vacuum ultraviolet radiation at the 15 th harmonic with echo-enabled harmonic generation technique, Phys. Rev. Accel. Beams 17, 070702 (2014).

[25] E. Allaria et al., High quality electron beams for high quality FEL, Proc. SPIE Int. Soc. Opt. Eng. 10237, 102370F (2017).

[26] E. L. Saldin, E. A. Schneidmiller, and M. V. Yurkov, Klystron instability of a relativistic electron beam in a bunch compressor, Nucl. Instrum. Methods Phys. Res., Sect. A 490, 1 (2002).

[27] J. Rosenzweig et al., Space-charge oscillations in a selfmodulated electron beam in multi-undulator free-electron lasers, Nucl. Instrum. Methods Phys Res., Sect. A 393, 376 (1997).

[28] Z. Huang, M. Borland, P. Emma, J. Wu, C. Limborg, G. Stupakov, and J. Welch, Suppression of microbunching instability in the linac coherent light source, Phys. Rev. Accel. Beams 7, 074401 (2004).

[29] S. Spampinati et al., Laser heater commissioning at an externally seeded free-electron laser, Phys. Rev. Accel. Beams 17, 120705 (2014).

[30] E. Allaria and G. De Ninno, Soft-X-Ray Coherent Radiation Using a Single-Cascade Free-Electron Laser, Phys. Rev. Lett. 99, 014801 (2007).

[31] Z. T. Zhao et al., First lasing of an echo-enabled harmonic generation free-electron laser, Nat. Photonics 6, 360 (2012).

[32] P. Rebernik et al., Coherent soft X-ray pulses from an echoenabled harmonic generation free-electron laser, Nat. Photonics 13, 555 (2019).

[33] G. Penco, E. Allaria, G. De Ninno, E. Ferrari, and L. Giannessi, Experimental Demonstration of Enhanced SelfAmplified Spontaneous Emission by an Optical Klystron, Phys. Rev. Lett. 114, 013901 (2015).

[34] G. Penco et al., Optimization of a high brightness photoinjector for a seeded FEL facility, J. Instrum. 8, P05015 (2013).

[35] M. Venturini et al., Microbunching instability in singlepass systems using a direct two-dimensional Vlasov solver, Phys. Rev. Accel. Beams 10, 104401 (2007). 
[36] S. Di Mitri, M. Cornacchia, C. Scafuri, and M. Sjöström, Electron beam optics and trajectory control in the Fermi free electron laser delivery system, Phys. Rev. Accel. Beams 15, 012802 (2012).

[37] S. Di Mitri and S. Spampinati, Microbunching instability study in a linac-driven free electron laser spreader beam line, Phys. Rev. Accel. Beams 20, 120701 (2017).

[38] P. Craievich et al., Implementation of Radio-Frequency Deflecting Devices for Comprehensive High-Energy Electron Beam Diagnosis, IEEE Trans. Nucl. Sci. 62, 210 (2015).

[39] G. Penco et al., Optical klystron enhancement to self amplified spontaneous emission at FERMI, Photonics 4, 15 (2017).

[40] E. Prat, Using the optical-klystron effect to increase and measure the intrinsic beam energy spread in free-electronlaser facilities, Phys. Rev. Accel. Beams 20, 040702 (2017).

[41] Y. Ding, P. Emma, Z. Huang, and V. Kumar, Optical klystron enhancement to self-amplified spontaneous emission free electron lasers, Phys. Rev. Accel. Beams 9, 070702 (2006).

[42] Y. Ding, P. Emma, Z. Huang, and V. Kumar, Erratum: Optical klystron enhancement to self-amplified spontaneous emission free electron lasers [Phys. Rev. Accel. Beams 9, 070702 (2006)], Phys. Rev. Accel. Beams 23, 019901 (2020).

[43] R. Bonifacio et al., Nuovo Cimento 13, 9 (1990).

[44] E. Roussel, Multicolor High-Gain Free-Electron Laser Driven by Seeded Microbunching Instability, Phys. Rev. Lett. 115, 214801 (2015).

[45] E. Ferrari, E. Allaria, W. Fawley, L. Giannessi, Z. Huang, G. Penco, and S. Spampinati, Impact of Non-Gaussian Electron Energy Heating upon the Performance of a Seeded Free-Electron Laser, Phys. Rev. Lett. 112, 114802 (2014).

[46] Z. Huang et al., Measurements of the linac coherent light source laser heater and its impact on the $\mathrm{x}$-ray free-electron laser performance, Phys. Rev. Accel. Beams 13, 020703 (2010).

[47] L. Pavlovic et al., in Proc. of the BIW 2010 Conference, Santa Fe, New Mexico, USA (2010), article TUPSM086.

[48] P. Finetti et al., Pulse Duration of Seeded Free-Electron Lasers, Phys. Rev. X 7, 021043 (2017).

[49] P. Craievich, S. DiMitri, M. Milloch, G. Penco, and F. Rossi, Modeling and experimental study to identify arrivaltime jitter sources in the presence of a magnetic chicane, Phys. Rev. Accel. Beams 16, 090401 (2013).

[50] E. Ferrari et al., Widely tunable two-colour seeded freeelectron laser source for resonant-pump resonant-probe magnetic scattering, Nat. Commun. 7, 10343 (2016).

[51] M. Zangrando et al., The photon analysis, delivery, and reduction system at the FERMI@Elettra free electron laser user facility, Rev. Sci. Instrum. 80, 113110 (2009).

[52] C. Svetina, D. Cocco, N. Mahne, L. Raimondi, E. Ferrari, and M. Zangrando, PRESTO, the on-line photon energy spectrometer at FERMI: design, features and commissioning results, J. Synchrotron Radiat. 23, 35 (2016).

[53] www.elettra.eu/lightsources/fermi.

[54] M. Žitnik et al., High Resolution Multiphoton Spectroscopy by a Tunable Free-Electron-Laser Light, Phys. Rev. Lett. 113, 193201 (2014).

[55] T. Takanashi et al., Time-Resolved Measurement of Interatomic Coulombic Decay Induced by Two-Photon Double Excitation of $\mathrm{Ne}_{2}$, Phys. Rev. Lett. 118, 033202 (2017).

[56] D. You et al., New Method for Measuring Angle-Resolved Phases in Photoemission, Phys. Rev. X 10, 031070 (2020).

[57] F. Bencivenga et al., Four-wave mixing experiments with extreme ultraviolet transient gratings, Nature (London) 520, 205 (2015).

[58] L. Foglia et al., First Evidence of Purely Extreme-Ultraviolet Four-Wave Mixing, Phys. Rev. Lett. 120, 263901 (2018). 